\title{
Theoretical Study of the Stability and Vibration Spectrum of $\mathrm{C}_{38} \mathrm{Cl}_{2}$
}

\author{
Zha Lin", Ma Guanhuai \\ College of Biology and Chemistry, Xingyi Normal University for Nationalities, Xingyi, China
}

\section{Email address:}

lincha9076@163.com (Zha Lin)

${ }^{*}$ Corresponding author

\section{To cite this article:}

Zha Lin, Ma Guanhuai. Theoretical Study of the Stability and Vibration Spectrum of $\mathrm{C}_{38} \mathrm{Cl}_{2}$. Science Discovery.

Vol. 9, No. 5, 2021, pp. 263-268. doi: 10.11648/j.sd.20210905.20

Received: September 9, 2021; Accepted: October 28, 2021; Published: November 5, 2021

\begin{abstract}
In this study, the theory of density functional was used to geometrically optimize the binary chlorine-derived isomers of $\mathrm{C}_{38}$ at the b3lyp/6-31g(d) level. Analysis of the reaction heat and HOMO-LUMO of the optimized structure shows that $\mathrm{C}_{38} \mathrm{Cl}_{2}$ is thermodynamically stable; The 1-8 added isomer $10-\mathrm{C}_{38} \mathrm{Cl}_{2}-2-37$ and The 1-4 added isomer $17-\mathrm{C}_{38} \mathrm{Cl}_{2}-12-27$ was the most stable among the molecules studied. Derivatization mostly occurs on the carbon atom with the most negative charge, which is generally the common vertex of three five membered rings. Chlorination derivatization reduces the HOMO energy level of the carbon cage, increases the HOMO-LUMO energy level gap of the carbon cage, and reduces the reaction activity of the carbon cage. The vibration frequency calculation shows that the addition mode and symmetry will affect the maximum vibration frequency and intensity of the structure. The strongest vibration is generally $\mathrm{C}-\mathrm{Cl}$ telescopic vibration. The higher the derived position symmetry, the greater the vibration intensity.
\end{abstract}

Keywords: $\mathrm{C}_{38} \mathrm{Cl}_{2}$, Stability, Vibration Spectrum, Density Functional Theory

\section{富勒烯 $\mathrm{C}_{38} \mathrm{Cl}_{2}$ 的稳定性和振动光谱的理论研究}

\section{查林*, 马关怀}

兴义民族师范学院生物与化学学院, 兴义, 中国

\section{邮箱}

lincha9076@163.com(查林)

摘要: 应用密度泛函理论在 $\mathrm{b} 31 \mathrm{yp} / 6-31 \mathrm{~g}(\mathrm{~d})$ 水平上对富勒烯 $\mathrm{C}_{38}$ 的二元氯化衍生异构体进行几何优化, 对优化结构的反应 热和前线轨道能级的分析表明, $\mathrm{C}_{38} \mathrm{Cl}_{2}$ 在热力学上是稳定的, 并且氯化衍生大多发生在电荷最负的碳原子上, 一般为 三个五元环共用顶点, 氯化衍生降低了碳笼的HOMO能级, 碳笼的HOMO-LUMO能级间隙得到增加, 降低了碳笼的反 应活性, 最稳定的异构体是 10 号母体上1-8加成的 $10-\mathrm{C}_{38} \mathrm{Cl}_{2}-2-37$ 和 17 号母体上1-4加成的 $17-\mathrm{C}_{38} \mathrm{Cl}_{2}-12-27$, 振动频率计算 发现, 加成方式和对称性会影响结构的最大振动频率及强度, 最强振动一般是 $\mathrm{C}-\mathrm{Cl}$ 伸缩振动, 衍生化位置对称性越高, 振动强度越大。

关键词: $\mathrm{C}_{38} \mathrm{Cl}_{2}$, 稳定性, 振动光谱, 密度泛函理论 


\section{1. 引言}

自从富勒烯 $\mathrm{C}_{60}[1]$ 发现以来, 其笼状结构和缺电子性 质使得富勒烯成为碳纳米材料领域的研究热点, Zeng等[2] 对近十年的富勒烯的研究进行统计, 认为富勒烯的研究重 点可能是用于太阳能电池的光能转换效率的提升以及医 疗应用。基于此, $\mathrm{YaO}$ 等[3]合成并分离了 $\mathrm{C}_{70}$ 的市基衍生物 2,5- $\left(\mathrm{PhCH}_{2}\right)_{2} \mathrm{C}_{70}$, 并作为钻钛矿太阳能电池器件, 提高光 电转换效率; Chen等[4]采用富勒烯的衍生物 N-甲基-2-戊 基[60]富勒烯吡啶烷用于反式钙钛矿太阳能电池器件, 同 样具有较高的电子传输速率。同时, 在医疗方面, 为改善 $\mathrm{C}_{60}$ 的溶解性, $\mathrm{Han}$ 等 [5]以 2-7二溴芴酮为基础, 合成含芴 基的富勒烯衍生物, Wang等[6]制备甲氧基富勒醇, 将其 与聚 $\beta$-环糊精进行超分子组装, 可以提高富勒烯的溶解性 和生物相容性, 培养基中添加了富勒醇, 能降低生物体活 性氧的水平, 有利于骨组织的再生。许多富勒烯内嵌物如 $\mathrm{Sc}_{2} \mathrm{O} @ \mathrm{C}_{78}[7] 、 \mathrm{Sc}_{2} \mathrm{C}_{2} @ \mathrm{Cs}(\mathrm{hept})-\mathrm{C}_{88}[8]$ 以及外接衍生物 $\mathrm{C}_{106} \mathrm{Cl}_{24} 、 \mathrm{C}_{108} \mathrm{Cl}_{12}$ [9]、 $\mathrm{C}_{78}(1)\left(\mathrm{CF}_{3}\right)(10-18)[10] 、 \mathrm{C}_{80} \mathrm{Cl}_{12}$ [11]、 $\mathrm{C}_{50} \mathrm{Cl}_{10}$ 以及 $\mathrm{C}_{78} \mathrm{Cl}_{16}$ [12]等得到合成和研究, 一般认为, 氯 化衍生是捕获、分离富勒烯的有效手段[13]。学者们前期 的研究能够为未来富勒烯及其衍生物的制备和表征提供 理论支持。
根据欧拉定理, 富勒烯 $\mathrm{C}_{38}$ 有 17 个异构体, 无法推测 谁是潜在的富勒烯衍生物母笼, 为系统搜索其可能的氯化 衍生异构体, 为未来其合成及鉴别提供理论支撑, 本工作 对富勒烯 $\mathrm{C}_{38}$ 的 17 个异构体的二元氯化衍生物采用密度泛 函理论中的b3lyp方法, 结合振动频率进行系统研究, 讨 论加成方式对其稳定性及振动光谱的影响。

\section{2. 计算过程}

部分富勒烯 $\mathrm{C}_{38}$ 的平面结构及碳原子编号如图1所示, 将氯原子加成在其中两个碳原子上, 以 $\mathrm{x}-\mathrm{C}_{38} \mathrm{Cl}_{2}-\mathrm{a}-\mathrm{b}$ 表示 $(\mathrm{x}$ 表示母体 $\mathrm{C}_{38}$ 的异构体编号; $\mathrm{a}, \mathrm{b}$ 分别表示加成的碳原子编 号）, 一共有1-2到1-8共七种加成方式（加成方式根据加 成碳原子之间最短 $\mathrm{C}-\mathrm{C}$ 键链上的碳原子数进行判定），如 13- $\mathrm{C}_{38} \mathrm{Cl}_{2}-4-35$ （图 1a）的加成位置为4、35 号碳原子, 加 成方式为1-6加成。由于异构体数目最多, 先用半经验方 法AM1对所有异构体进行优化, 根据不同加成方式进行篮 选, 对每种母体的每种加成方式中能量最低的总共119个 $\mathrm{C}_{38} \mathrm{Cl}_{2}$ 异构体, 采用b3lyp 方法结合 6-31g(d)基组对其进行 更高精度的计算, 最后对每种母体中能量最低的 $\mathrm{C}_{38} \mathrm{Cl}_{2}$ 异 构体进行振动频率分析。

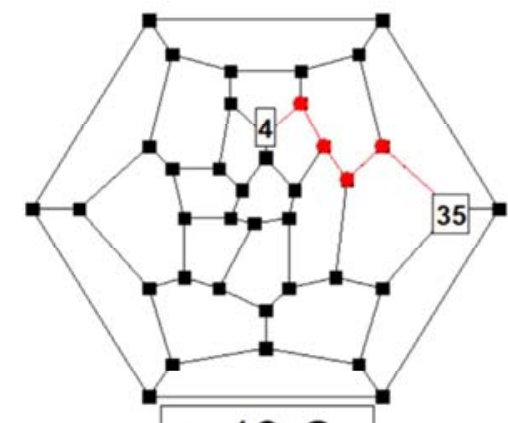

a: $13-C_{38}$

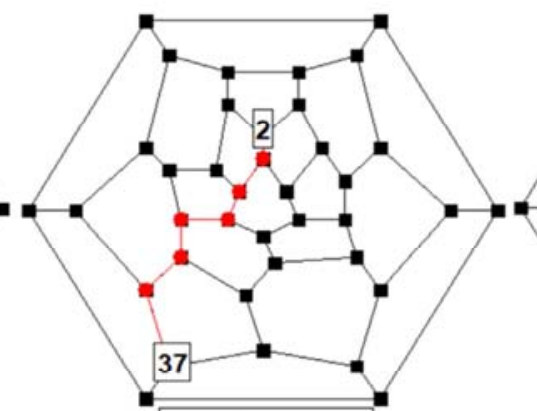

b: $10-C_{38}$

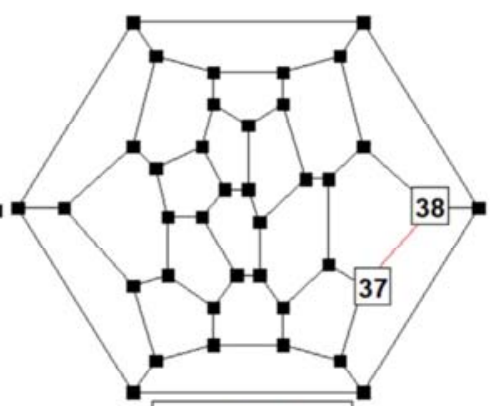

c: $8-\mathrm{C}_{38}$

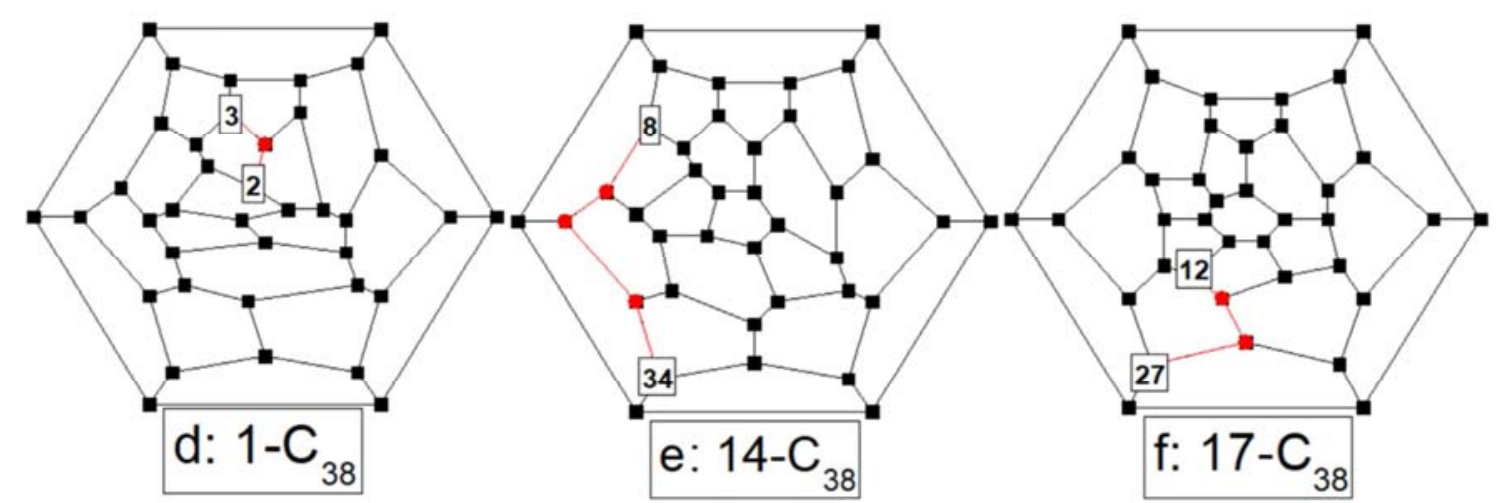

图1 部分 $\mathrm{C}_{38}$ 的平面结构及加成位置图。

\section{3. 结果与讨论}

在 $\mathrm{C}_{38}$ 的 17 个异构体上进行氯化衍生的异构体在 b3lyp $/ 6-31 \mathrm{~g}(\mathrm{~d})$ 水平上计算出来的能量最低的 $\mathrm{C}_{38} \mathrm{Cl}_{2}$ 异构体 的加成方式、反应热 $\Delta \mathrm{H}\left(\Delta \mathrm{H}=\mathrm{E}\left(\mathrm{C}_{38} \mathrm{Cl}_{2}\right)-\mathrm{E}\left(\mathrm{C}_{38}\right)-\mathrm{E}\left(\mathrm{Cl}_{2}\right)\right)$
以及相对能量 $\Delta \mathrm{E}$ (相对能量最低的17- $\mathrm{C}_{38} \mathrm{Cl}_{2}-12-27$ ) 列于 表1。

从反应热可以判断衍生物的稳定性, 反应热越负, 结 构越稳定 [14], 所计算的所有衍生物的反应热均为负值, 表明 $\mathrm{C}_{38} \mathrm{Cl}_{2}$ 是热力学稳定的, 其中反应热最负的是 $10-$ 
$\mathrm{C}_{38} \mathrm{Cl}_{2}-2-37$ 。但是从绝对能量分析, 能量最低的反而是 $17-\mathrm{C}_{38} \mathrm{Cl}_{2}-12-27$, 其次才是 $10-\mathrm{C}_{38} \mathrm{Cl}_{2}-2-37$, 考虑到其母体 $17-\mathrm{C}_{38}$ 是所有的母体中能量最低的结构, 可以预测, 二者 均可能是潜在的二元氯化衍生结构。每个母体的能量最低 的衍生物的加成方式不一, 从1-2到1-8加成均存在, 说明 对于 $\mathrm{C}_{38}$ 而言, 无法预测其加成模式, 可能需要根据其几 何结构或者电子结构进行判断。

HOMO-LUMO能级及间隙与稳定性有一定关系, 为 便于比较, 将所计算的 119 种 $\mathrm{C}_{38} \mathrm{Cl}_{2}$ 异构体的 $\mathrm{HOMO}$ 、 LUMO 能级以及能级间隙相对于母体碳笼的相对值的统 计结果列于图 2 。和母体富勒烯碳笼 $\mathrm{C}_{38}$ 相比, 衍生物的 HOMO 大多低于母体（图2a），降幅最大的是 1-4加成的 6- $\mathrm{C}_{38} \mathrm{Cl}_{2}-9-30$, 其次是所有异构体中能量最低的 17$\mathrm{C}_{38} \mathrm{Cl}_{2}-12-27$, 反应热最负的 $10-\mathrm{C}_{38} \mathrm{Cl}_{2}-2-37$ 排第三。衍生 物的LUMO能级和母体富勒烯碳笼 $\mathrm{C}_{38}$ 相比有升高、有降 低 (图2b), 增幅最大的是反应热最负的 $10-\mathrm{C}_{38} \mathrm{Cl}_{2}-2-37$ 。 衍生物的HOMO-LUMO能级间隙 (Gap) 值和母体富勒烯 碳笼 $\mathrm{C}_{38}$ 相比, 大多数增加（图 $2 \mathrm{c}$ ), Gap值最大的是反应 热最负的 $10-\mathrm{C}_{38} \mathrm{Cl}_{2}-2-37$, 其次是 $17-\mathrm{C}_{38} \mathrm{Cl}_{2}-12-27$, 可见氯 化衍生降低了碳笼的反应活性, 最可能的衍生化异构体应 该是 1-4 加成的 17- $\mathrm{C}_{38} \mathrm{Cl}_{2}-12-27$ 以及 1-8 加成的 10$\mathrm{C}_{38} \mathrm{Cl}_{2}-2-37$ 。

表 $1 \mathrm{C}_{38} \mathrm{Cl}_{2}$ 异构体的加成方式、反应热以及相对能量。

\begin{tabular}{llll}
\hline 加成方式 & 异构体 & $\begin{array}{l}\text { 反应热 } \\
\text { Addition mode }\end{array}$ & $\begin{array}{l}\text { 相对能量 } \\
\text { Isomer }\end{array}$ \\
\hline $1-3$ & $1-\mathrm{C}_{38} \mathrm{Cl}_{2}-2-3$ & -322.6 & 377.9 \\
$1-8$ & $2-\mathrm{C}_{38} \mathrm{Cl}_{2}-2-37$ & -358.2 & 462.6 \\
$1-7$ & $3-\mathrm{C}_{38} \mathrm{Cl}_{2}-1-33$ & -327.4 & 206.5 \\
$1-2$ & $4-\mathrm{C}_{38} \mathrm{Cl}_{2}-37-38$ & -307.1 & 289.0 \\
$1-8$ & $5-\mathrm{C}_{38} \mathrm{Cl}_{2}-1-38$ & -304.4 & 139.1 \\
$1-2$ & $6-\mathrm{C}_{38} \mathrm{Cl}_{2}-1-4$ & -282.7 & 216.2 \\
$1-8$ & $7-\mathrm{C}_{38} \mathrm{Cl}_{2}-3-37$ & -319.4 & 240.9 \\
$1-2$ & $8-\mathrm{C}_{38} \mathrm{Cl}_{2}-37-38$ & -305.6 & 123.6 \\
$1-4$ & $9-\mathrm{C}_{38} \mathrm{Cl}_{2}-1-11$ & -296.0 & 270.5 \\
$1-8$ & $10-\mathrm{C}_{38} \mathrm{Cl}_{2}-2-37$ & -365.3 & 22.7 \\
$1-5$ & $11-\mathrm{C}_{38} \mathrm{Cl}_{2}-5-9$ & -275.2 & 187.3 \\
$1-7$ & $12-\mathrm{C}_{38} \mathrm{Cl}_{2}-2-37$ & -325.6 & 281.2 \\
$1-6$ & $13-\mathrm{C}_{38} \mathrm{Cl}_{2}-4-35$ & -315.5 & 34.6 \\
$1-5$ & $14-\mathrm{C}_{38} \mathrm{Cl}_{2}-8-34$ & -295.2 & 86.8 \\
$1-8$ & $15-\mathrm{C}_{38} \mathrm{Cl}_{2}-7-32$ & -323.4 & 184.1 \\
$1-6$ & $16-\mathrm{C}_{38} \mathrm{Cl}_{2}-19-26$ & -289.2 & 105.3 \\
$1-4$ & $17-\mathrm{C}_{38} \mathrm{Cl}_{2}-12-27$ & -270.8 & 0.0 \\
\hline
\end{tabular}

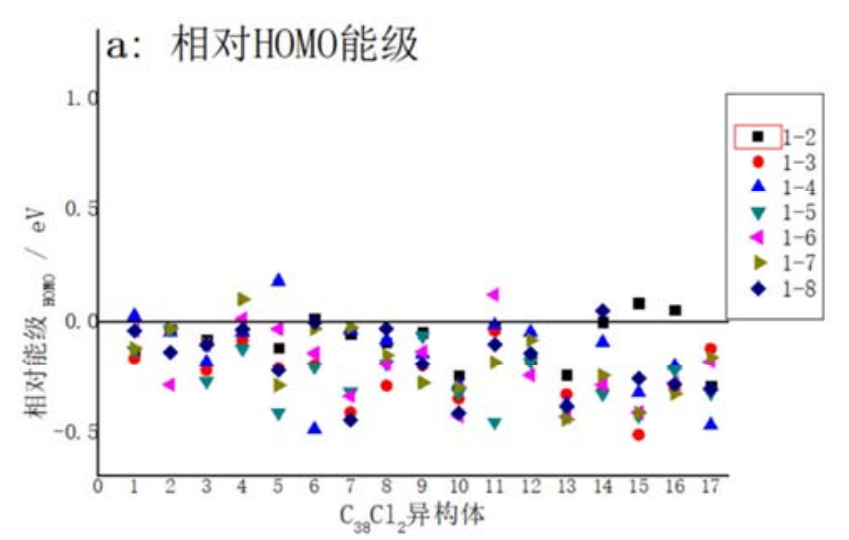

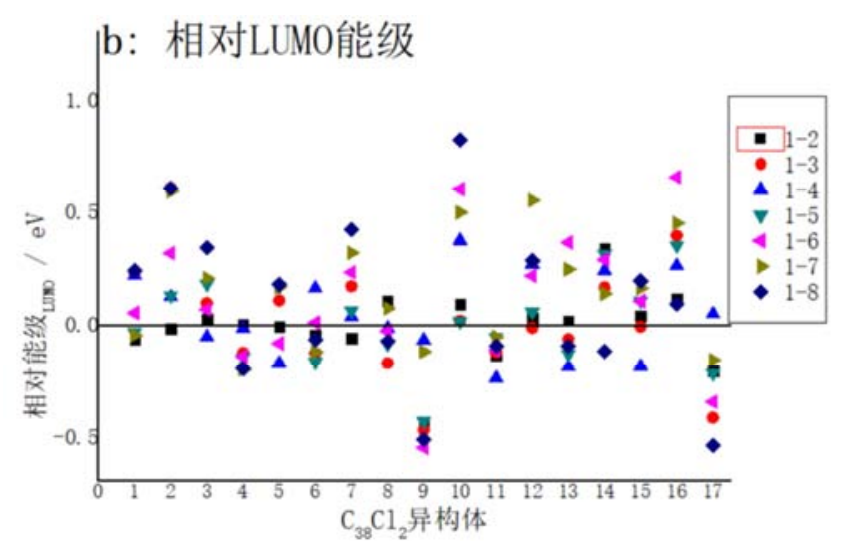

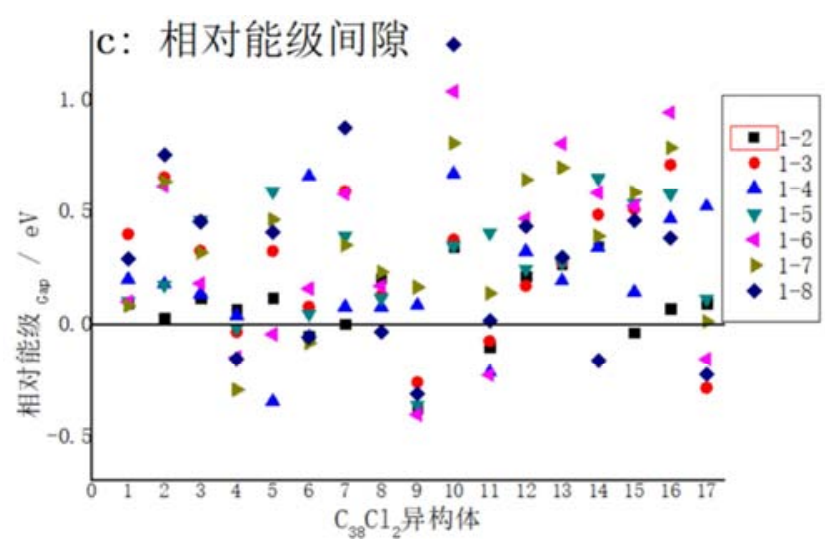

图 $2 \mathrm{C}_{38} \mathrm{Cl}_{2}$ 的相对HOMO-LUMO能级。

对富勒烯母体的结构分析发现, 发现最稳定的二 元氯化衍生大多发生在电荷最负的碳原子上, 对于母 体 $\mathrm{C}_{38}$ 而言, 一般电荷最负的位置为三个五元环共用顶 点, 例如 10- $\mathrm{C}_{38}$ （图 1b) 上 2 号碳原子为三个五元环共 用顶点, 周围分布 9 个碳原子, 一般称 $\mathrm{C}_{10}$ 顶点, 反应 热最负的 $10-\mathrm{C}_{38} \mathrm{Cl}_{2}-2-37$ 的 $2 、 37$ 号碳原子是母体 $10-\mathrm{C}_{38}$ 上唯一的两个 $\mathrm{C}_{10}$ 顶点。比较不同碳笼的最稳定衍生异 构体, 氯原子在锁定一个 $\mathrm{C}_{10}$ 顶点后, 另一个氯原子则 在附近寻找是否存在 $\mathrm{C}_{10}$, 如 $8-\mathrm{C}_{38}$ (图 $1 \mathrm{c}$ ) 和 1- $\mathrm{C}_{38}$ (图 $1 \mathrm{~d}$ ) 存在相邻或者间隔的 $\mathrm{C}_{10}$ 顶点, 因此发生比较近的 1-2和 1-3 加成。如附近位置无 $\mathrm{C}_{10}$ 顶点, 则一般在更远 的 1-7, 1-8加成位置寻求另外的 $\mathrm{C}_{10}$ 进行衍生, 如最远 位置没有 $\mathrm{C}_{10}$, 则在 1-5, 1-6位置在尽量保持异构体对 称性的基础上进行加成, 例如 $14-\mathrm{C}_{38}$ (图1e) 有唯一一 个 $\mathrm{C}_{10}$ 顶点为 34 号碳原子, 另外在 1-5 位置寻求 8 号碳原 子进行加成。需要注意的是, $17-\mathrm{C}_{38}$ (图1f) 由于没有 $\mathrm{C}_{10}$ 点, 两个 $\mathrm{C}_{556}$ (两个五元环和一个六元环共用顶点) 的 1-4 环内加成成为最可能的衍生化位置, 17$\mathrm{C}_{38} \mathrm{Cl}_{2}$-12-27为所有异构体中能量最低的衍生物, 另一 个发生 1-4加成的 $9-\mathrm{C}_{38}$ 虽然存在多个 $\mathrm{C}_{10}$ 顶点, 但母体 为 $\mathrm{D}_{3}$ 对称性, 如在 $\mathrm{C}_{10}$ 位置进行加成, 破坏对称性, 所 以同样选择两个 $\mathrm{C}_{556}$ 的1-4环内加成。

为验证衍生物 $\mathrm{C}_{38} \mathrm{Cl}_{2}$ 在势能面上的属性, 了解不同加 成位置对衍生物振动形式的影响, 对 17 种母体中能量最低 的衍生异构体进行振动频率分析, 衍生物的最大振动频率 (强度最大对应的频率) 及强度列于表 2 中。 
表 $2 \mathrm{C}_{38} \mathrm{Cl}_{2}$ 异构体的最大振动频率及强度。

\begin{tabular}{llll}
\hline $\begin{array}{l}\text { 加成方式 } \\
\text { Addition mode }\end{array}$ & $\begin{array}{l}\text { 异构体 } \\
\text { Isomer }\end{array}$ & $\begin{array}{l}\text { 最大振动频率 } \\
\text { Frequency } / \mathbf{c m}^{-1}\end{array}$ & $\begin{array}{l}\text { 最大振动强度 } \\
\text { Intensity }\end{array}$ \\
\hline $1-3$ & $1-\mathrm{C}_{38} \mathrm{Cl}_{2}-2-3$ & 921.0 & 212.0 \\
$1-8$ & $2-\mathrm{C}_{38} \mathrm{Cl}_{2}-2-37$ & 839.4 & 467.7 \\
$1-7$ & $3-\mathrm{C}_{38} \mathrm{Cl}_{2}-1-33$ & 821.9 & 177.8 \\
$1-2$ & $4-\mathrm{C}_{38} \mathrm{Cl}_{2}-37-38$ & 902.4 & 106.1 \\
$1-8$ & $5-\mathrm{C}_{38} \mathrm{Cl}_{2}-1-38$ & 829.1 & 480.5 \\
$1-2$ & $6-\mathrm{C}_{38} \mathrm{Cl}_{2}-1-4$ & 764.1 & 98.9 \\
$1-8$ & $7-\mathrm{C}_{38} \mathrm{Cl}_{2}-3-37$ & 830.9 & 550.2 \\
$1-2$ & $8-\mathrm{C}_{38} \mathrm{Cl}_{2}-37-38$ & 767.3 & 122.2 \\
$1-4$ & $9-\mathrm{C}_{38} \mathrm{Cl}_{2}-1-11$ & 894.4 & 188.5 \\
$1-8$ & $10-\mathrm{C}_{38} \mathrm{Cl}_{2}-2-37$ & 837.2 & 431.7 \\
$1-5$ & $11-\mathrm{C}_{38} \mathrm{Cl}_{2}-5-9$ & 817.3 & 180.1 \\
$1-7$ & $12-\mathrm{C}_{38} \mathrm{Cl}_{2}-2-37$ & 837.2 & 292.7 \\
$1-6$ & $13-\mathrm{C}_{38} \mathrm{Cl}_{2}-4-35$ & 819.6 & 168.1 \\
$1-5$ & $14-\mathrm{C}_{38} \mathrm{Cl}_{2}-8-34$ & 820.0 & 155.1 \\
$1-8$ & $15-\mathrm{C}_{38} \mathrm{Cl}_{2}-7-32$ & 833.3 & 402.3 \\
$1-6$ & $16-\mathrm{C}_{38} \mathrm{Cl}_{2}-19-26$ & 815.9 & 152.8 \\
$1-4$ & $17-\mathrm{C}_{38} \mathrm{Cl}_{2}-12-27$ & 814.1 & 234.2 \\
\hline
\end{tabular}

每种衍生物均有 40 个原子, 有 114 种振动形式, 对衍 生物进行振动频率分析时, 没有发现虚频, 表明这些结构 均为势能面上的极小点, 是稳定结构 [15]。衍生化异构体 的最小频率是外接氯原子的面内外振动, 位置不一, 振动 频率也有差异。由于存在氯原子, 衍生物在 $200 \mathrm{~cm}^{-1}$ 以下 有四个振动频率, 分别是 $\mathrm{C}-\mathrm{Cl}$ 的面内（剪式和摇摆）及面 外振动（摇摆及扭曲），1-8加成的异构体由于两个加成 氯原子几乎在碳笼两端, 无法准确判断其振动模式, 1-2 加成的异构体的四个最小振动频率从小到大依次对应面 外扭曲、面外摇摆、面内摇摆和面内剪式振动, 1-3到1-7 加成的异构体除 1-4加成的 $9-\mathrm{C}_{38} \mathrm{Cl}_{2}-1-11$ 外最小振动频率
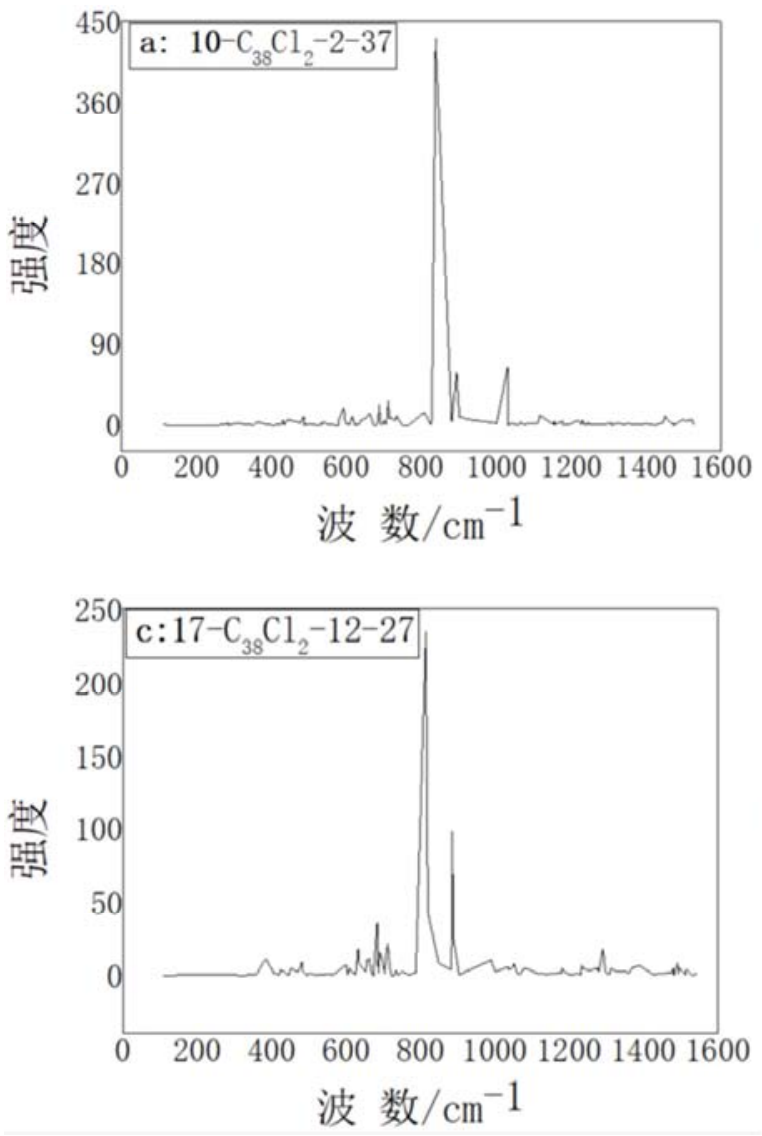

均为面内剪式振动, 值得注意的是 $12-\mathrm{C}_{38} \mathrm{Cl}_{2}-2-37,13-$ $\mathrm{C}_{38} \mathrm{Cl}_{2}-4-35$ 的加成位置的对称性导致面外扭曲振动没有 导致偶极矩变化, 没有红外吸收 (强度为 0 )。部分 $\mathrm{C}_{38} \mathrm{Cl}_{2}$ 异构体的振动光谱绘制于图3中, 对富勒烯 $\mathrm{C}_{38} \mathrm{Cl}_{2}$ 振动谱图 而言, 谱图简单, 大多在 $810 \sim 840 \mathrm{~cm}^{-1}$ 左右附近有一强吸 收带, 最强振动一般是 $\mathrm{C}-\mathrm{Cl}$ 伸缩振动。从表2看出, 所计 算的1-8加成的衍生物的最强振动强度最强, 一般在 400 以 上, 这是因为氯原子几乎对称分布在碳笼两侧, 相当于两 个C-Cl收缩振动的累加, 因此强度最强, 最大振动频率一 般在830 $840 \mathrm{~cm}^{-1}$ 之间, 一般为一尖峰, 如反应热最负的 衍生物 10- $\mathrm{C}_{38} \mathrm{Cl}_{2}-2-37$ (图3a) 的强度为431.7。1-2加成的 8- $\mathrm{C}_{38} \mathrm{Cl}_{2}-37-38$ (图3b) 的峰发生分裂为两组峰, $600 \sim 800$ $\mathrm{cm}^{-1}$ 为一宽峰, $900 \mathrm{~cm}^{-1}$ 左右为一尖峰, 两组峰的强度均 较小，在 $100 \sim 120$ 上下; 最稳定衍生物 1-4加成的 17$\mathrm{C}_{38} \mathrm{Cl}_{2}-12-27$ (图3c) 同样分裂为两组, 但峰型较尖, 强度 稍大，在200左右。1-5加成的14- $\mathrm{C}_{38} \mathrm{Cl}_{2}-8-34$ (图3d)、1-6 加成的13- $\mathrm{C}_{38} \mathrm{Cl}_{2}-4-35$ （图3e）、1-7加成的3- $\mathrm{C}_{38} \mathrm{Cl}_{2}-1-33$ 的最强峰在 $810 \sim 840 \mathrm{~cm}^{-1}$ 之间, 强度介于1-2加成和1-4加 成两者之间, 强度大概在 $150 \sim 180$ 左右, 但1-7加成的12$\mathrm{C}_{38} \mathrm{Cl}_{2}-2-37$ 是个例外, 其2、37号碳原子几乎在碳笼两端, 强度较大。1-3加成的1- $\mathrm{C}_{38} \mathrm{Cl}_{2}-2-3$ （图3f）的振动图则与 其他不同, 分裂为三组, 在 $730 \mathrm{~cm}-1 、 810 \mathrm{~cm}^{-1} 、 920 \mathrm{~cm}^{-1}$ 附近分别有三组, 强度依次增大。相同加成方式的谱图形 状大体相同, 异构体的频率分析可作为鉴别富勒烯 $\mathrm{C}_{38} \mathrm{Cl}_{2}$ 的不同加成位置衍生物的一个依据。
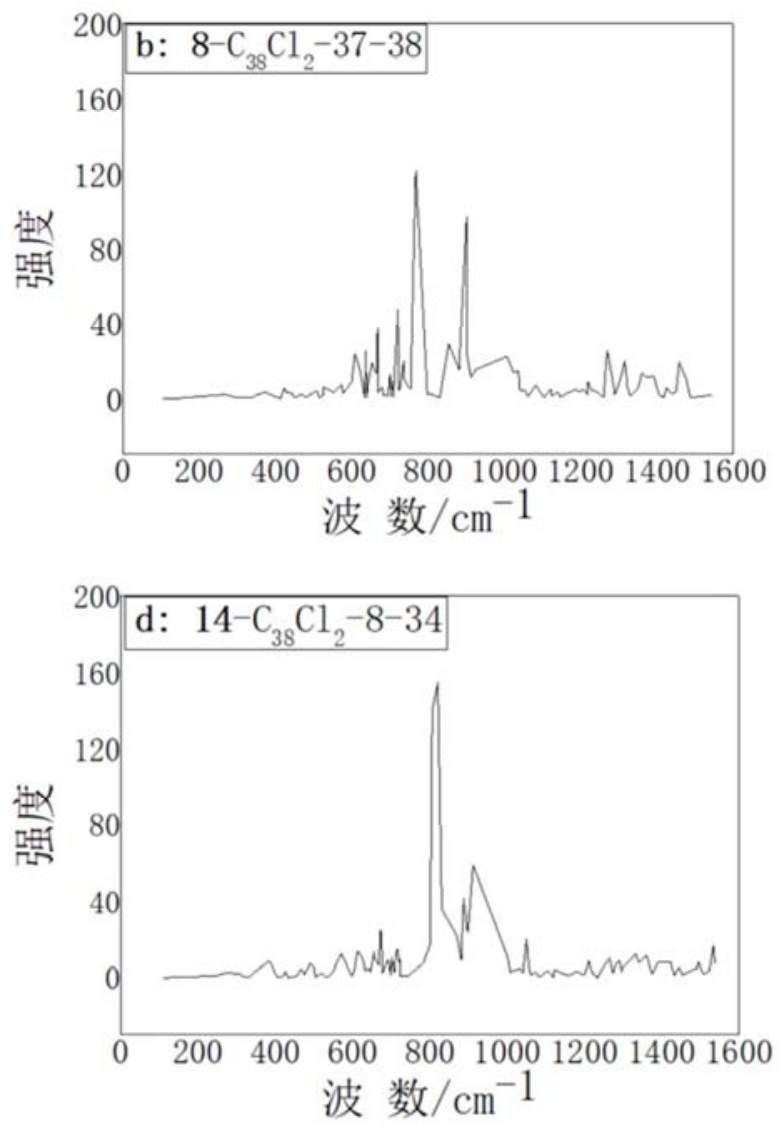

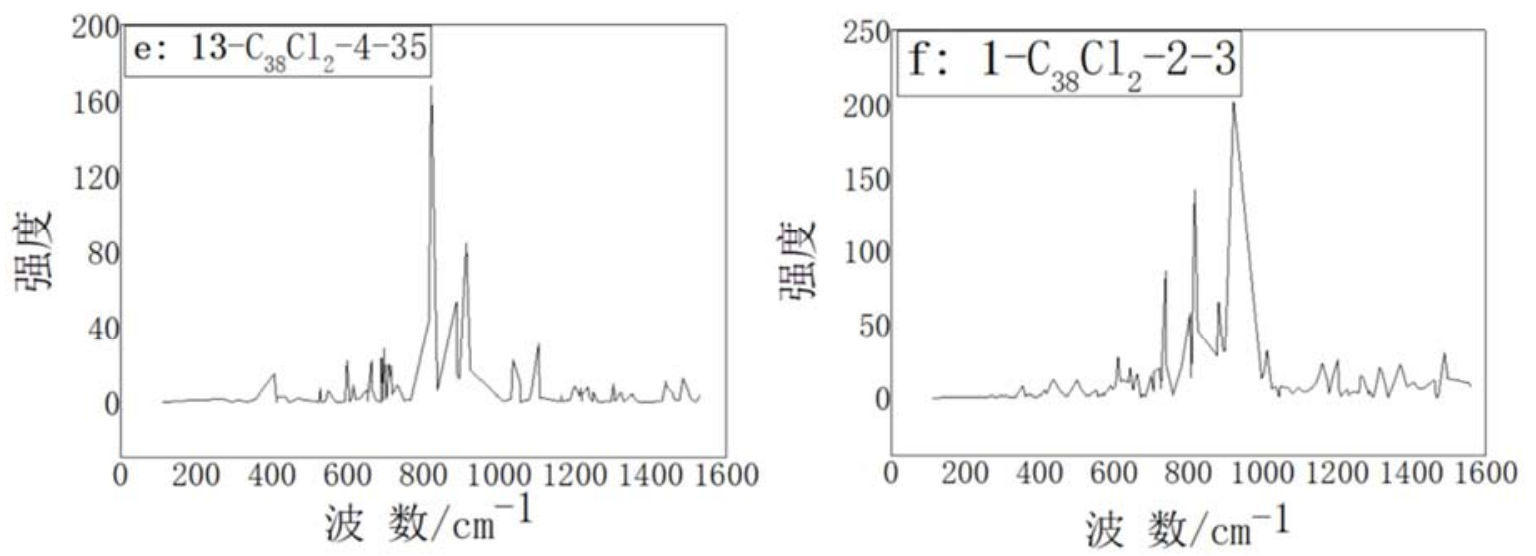

图3 部分 $\mathrm{C}_{38} \mathrm{Cl}_{2}$ 的振动光谱。

\section{4. 结论}

应用密度泛函理论对富勒烯 $\mathrm{C}_{38}$ 的二元氯化衍生异构 体进行几何优化, 对优化结构的反应热、前线轨道能级及 能级间隙的分析表明, $\mathrm{C}_{38} \mathrm{Cl}_{2}$ 在热力学上是稳定的, 最容 易加成的位置是三个五元环共用顶点, 衍生化倾向于发生 在电荷较负的位置, 10 号母体上1-8加成的 $10-\mathrm{C}_{38} \mathrm{Cl}_{2}-2-37$ 反应热最负, 17 号母体上1-4加成的 $17-\mathrm{C}_{38} \mathrm{Cl}_{2}-12-27$ 的相对 能量最小, 二者均为最可能的衍生化产物, 应该最为稳定。 通过振动频率计算发现，加成位置会影响结构的最大振动 频率及强度, 1-8加成的振动强度较大, 为一尖峰, 1-2加 成振动强度最弱, 并且和其他加成方式一样, 峰形分裂, 异构体的频率分析可作为鉴别 $\mathrm{C}_{38} \mathrm{Cl}_{2}$ 的不同加成衍生物的 一个依据。本实验系统搜索两个氯原子的各种加成模式, 得到的结构虽然只有两个活性碳原子受到加成, 但这些结 构可以为进一步加成形成更高衍生化提供了基础。

\section{致谢}

本文为黔西南州科技基金（2014-8）；兴义民族师范 学院教授基金（17XYJS11）的阶段性成果之一。

\section{参考文献}

[1] KROTO H W, HEATH J R, O'BRIEN S C, et al. $\mathrm{C}_{60}$ : Buckminsterfullerene[J]. Nature, 1985, 318: 162-163.

[2] ZENG S X(曾硕勋), FENG M(冯敏), JING L(姜玲). Research hot spots and fronts mining of material science: A case study of fullerence[J]. Materlals China(中国材料进展), 2019, 38(2): 161-166.

[3] YAO C R(姚春瑞), LI S H(李姝慧), XING Z(邢舟), et al. Synthesis of C70 benzyl derivative and its application in perovskite solar cells[J]. Journal of Xiamen University(Natural Science)(厦门大学学报 (自然科学版)), 2020, 59(1): 19-25.
[4] CHEN R(陈瑞), WANG W(王维), BU T L(卜童乐), et al. Low-cost fullerence derivative as an efficient electron transport Layer for planar perovskite solar cells[J]. Acta Physico-Chimica Sinica(物理化学学报), 2019, 35(4): 401-407.

[5] HAN L(韩露), LIU F L(刘奉丽), DING M Y(丁敏源), et al. Synthesis of novel [60]Fullerene derivatives containing flourene[J]. Chinese Journal of Synthetic Chemistry(合成化 学), 2016, 24(3): 219-222.

[6] WANG X J(王先津), LI J L(李井龙), SAI T(赛霆), et al. Synthesis of methoxy fullerol and Its supramolecular assembly and characterization[J]. Chemistry \& Bioengineering(化学与生物工程), 2017, 34(5): 40-45.

[7] ZHAO P, LI M Y, GUO Y J, et al. Single Step Stone-Wales Transformation Linking Two Thermodynamically Stable $\mathrm{Sc}_{2} \mathrm{O} @ \mathrm{C}_{78}$ Isomers[J]. Inorganic Chemistry, 2016, 55: 2220-2226.

[8] CHEN C H, ABELla L, CERON M R, et al. Zigzag $\mathrm{Sc}_{2} \mathrm{C}_{2}$ carbide cluster inside a [88] fullerene cage with one heptagon, $\mathrm{Sc}_{2} \mathrm{C}_{2} @ \mathrm{Cs}$ (hept)- $\mathrm{C}_{88}$ : A kinetically trapped fullerene formed by $\mathrm{C}_{2}$ insertion?[J]. Journal of the American Chemical Society, 2016, 138: 13030-13037.

[9] WANG S, YANG S F, KEMNITZ E, et al. New giant fullerenes identified as chloro derivatives: Isolated Pentagon-Rule $\mathrm{C}_{108}{ }^{(1771)} \mathrm{C}_{112}$ and $\mathrm{C}_{106}{ }^{(1155)} \mathrm{Cl}_{24}$ as well as nonclassical $\mathrm{C}_{104} \mathrm{Cl}_{24}[\mathrm{~J}]$. Inorganic Chemistry, 2016, 55: 5741-5743.

[10] KOSAYA M P, FRITZ M A, BROTSMAN V A, et al. Synthesis, Isolation and structure of trifluoromethylated fullerene $\mathrm{D}_{3}-\mathrm{C}_{78}, \mathrm{C}_{78}(1)\left(\mathrm{CF}_{3}\right) 10-18[\mathrm{~J}]$. Chemistry-An Asian Journal, 2016, 11: 1000-1003.

[11] SHEN H T(沈洪涛), WANG D L(王东来), SUN X P(孙晓 萍), et al. Theoretical Study on Fullerene $\mathrm{C}_{80}$ 's Derivatives $\mathrm{C}_{80} \mathrm{X}_{12}(\mathrm{X}=\mathrm{H}, \mathrm{F}, \mathrm{Cl}, \mathrm{Br})[\mathrm{J}]$. Journal of Northeastern University(Natural Science) (东北大学学报 (自然科学版)), 2010, 32(2): 217-220.

[12] ZHONG Y Y(钟园园), TIAN H R(田寒荵), YAO Y Y(姚阳 榕), et al. Capture and structure characterization of ${ }^{\# 24106} \mathrm{C}_{78} \mathrm{Cl}_{6}$ in carbon $\operatorname{arc}[\mathrm{J}]$. Journal of Xiamen University(Natural Science)(厦门大学学报 (自然科学版)), 2019, 58(1): 27-33. 
[13] YIN F H(尹凡华),TAN K(谭凯).Density Functional Theory Study on the Formation Mechanism of Isolated-Pentagon-Rule $\quad \mathrm{C}_{100}(417) \mathrm{Cl}_{28}[\mathrm{~J}] . \quad$ Acta Physico-Chimica Sinica(物理化学学报),2018,34(3):256-262.

[14] Liu X S (刘学森), Lei D (雷丹), Gan L H(甘利华). Computational study on trimetallic nitride fullerenes $\mathrm{MSc}_{2}$ $\mathrm{N} @ \mathrm{C}_{76}$. Sci Sin Chim（中国科学）, 2016, 46: 915-922.
[15] SHEN H T(沈洪涛),IU L Y(刘丽影),WANG D L(王东来), et al. Theoretical study on fullerene $\mathrm{C}_{72}$ 's derivatives $\mathrm{C}_{72} \mathrm{X}_{4}(\mathrm{X}=\mathrm{H}, \mathrm{F}, \mathrm{Cl})[\mathrm{J}]$.Chemical Research and Application(化学 研究与应用),2012, 24(8): 1202-1206. 